\title{
Prácticas de Matemáticas 2: de la clase tradicional a la clase inversa
}

Anna Vidal Melóa, Francisco J. Boigues Planes ${ }^{b}$ y Vicente D. Estruch ${ }^{c}$

Grup d'Innovació Educativa i recerca en Matèries Científiques. Universitat Politècnica de ValènciaCampus de Gandia. Departament de Matemàtica Aplicada. aavidal@mat.upv.es, bfraboipl@mat.upv.es y ${ }^{\mathrm{c}}$ vdestruc@mat.upv.es.

\begin{abstract}
In honour of Confucius when he said "I hear and I forget. I see and I remember. I do and I understand" and through Universitat Politècnica de València efforts to promote active methodologies as a starting point in the construction of learning processes, the so-called Flipped Teaching technique or reversed class has gained a significant importance in our subjects. The improvement lies in several aspects. The work commonly done at home, such as problem solving, has shifted from home to the classrooms. Moreover, the teacher acts as a mentor rather than a purveyor of knowledge. Through this methodology, the relevance of the lecturer decreases placing emphasis on the student and the learning process. Thus, students can prepare the theoretical part in advance and take the opportunity to conduct practical activities and teamwork as well as to clarify doubts during on-site classes in real time. Collaboration between students and professors guidance play a decisive role in the teaching and learning process. In this paper, we will outline the carried out experience to reverse the lessons involved in the subject "Mathematics 2" belonging to the first year of the Degree in Telecommunication, Sound and Image Systems Engineering as a result of two projects specialized in educational innovation.
\end{abstract}

Keywords: Flipped Teaching, active methodologies, team work, Mathematics.

\footnotetext{
Resumen

Haciendo honor a Confucio cuando dijo "Me lo contaron y lo olvidé, lo vi y lo entendí, lo hice y lo aprendi', y gracias a los esfuerzos de la Universitat Politècnica de València por potenciar las metodologías activas como punto de partida en la construcción de los aprendizajes, el Flipped Teaching o clase inversa está tomando una especial relevancia en nuestras asignaturas, dado que da gran importancia al trabajo que comúnmente se hacía en casa, como era la resolución de problemas, y que ahora se aborda en el aula, donde el papel del profesor es el de orientador más que de transmisor de conocimientos. En esta metodología se rebaja el protagonismo del docente para hacer más énfasis en el del alumno y su aprendizaje. Los estudiantes pueden preparar la teoría en casa con anterioridad, aprovechando la clase
} 
presencial para realizar actividades más prácticas, trabajos en grupo y aclarar las dudas que les vayan surgiendo en la resolución de problemas en tiempo real, a través de la colaboración con sus compañeros de equipo junto con las orientaciones del profesor, constituyendo todo este proceso una parte muy significativa en su aprendizaje. En este trabajo, describiremos la experiencia llevada a cabo para invertir la clase en la asignatura Matemáticas 2 del primer año del Grado en Ingeniería de Sistemas de Telecomunicación, Sonido e Imagen, resultado de dos proyectos de innovación educativa.

Palabras clave: Flipped Teaching, metodologías activas, trabajo en grupo, Matemáticas.

\section{Introducción}

La Universitat Politècnica de València (UPV) impulsa la aplicación de metodologías docentes activas que potencien el trabajo en equipo, la resolución de problemas, la realización de prácticas, etc. Es el punto de partida en la construcción y puesta en prática de nuevas formas de aprender, como es el caso del Flipped Teaching (FT) o clase inversa. Este nuevo enfoque del aprendizaje supone que el estudiante trabaje en casa la parte teórica, de manera autónoma, mediante materiales seleccionados o creados por el profesor (vídeos, apuntes, etc), dedicando la clase presencial a realizar prácticas, actividades grupales y a aclarar las dudas que vayan surgiendo en la resolución de problemas. En el FT, resulta fundamental interacción colaborativa entre compañeros y, cómo no, el trabajo del profesor, cuyo papel será principalmente el de orientar o guíar en el proceso de aprendizaje. Desde hace varios años el FT está tomando una especial relevancia en la UPV, aumentando año tras año el número de profesores y de asignaturas que adoptan esta metodología.

Gracias al apoyo del Vicerrectorado de Estudios, Calidad y Acreditación de la UPV, dos Proyectos de Innovación Docente han facilitado a los miembros del "Grup d'Innovació Educativa i Recerca en Matèries Científiques" (GIERMAC) abordar y hacer realidad el enfoque FT en el aula. El primer paso fué analizar la posible introducción de esta nueva metodología en nuestras asignaturas, con el PIME "Estudio sobre la aplicación del Flip Teaching en asignaturas de Matemáticas y Física" (convocatoria 2015-2016). En segundo lugar se han estudiado los resultados de aplicar la metodología FT, con el PIME "Puesta en marcha de diversas experiencias con el enfoque FT en asignaturas de Matemáticas y Física" (convocatoria 2016-2017). En ambos proyectos se ha contado con un equipo interdisciplinar de profesores (Departamento de Matemática Aplicada y Departamento de Física Aplicada), desarrollándose en asignaturas de diversos Grados (Vidal et al, 2016).

Matemáticas 2, asignatura de primer curso del Grado en Ingeniería de Sistemas de Telecomunicación, Sonido e Imagen (Campus de Gandia-UPV) es una de las asignaturas

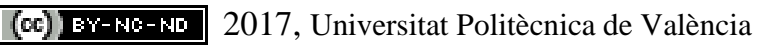


involucradas en estos proyectos. En este trabajo se presenta la experiencia y la labor docente realizada desde el curso 2015-2016 en dicha asignatura.

En el FT son importantes, además de la creación y el uso de vídeos, las actividades que los alumnos desarrollan en el aula. A la hora de diseñar actividades, en conveniente tener presente la denominada pirámide del aprendizaje de Blair que mostramos en la Figura 1. Esta pirámide plasma la conocida idea de Confucio: "Me lo contaron y lo olvidé, lo ví y lo entendí, lo hice y lo aprendí", y resume cómo aprenden los estudiantes de forma más efectiva, así como el impacto que tienen las diversas actividades en dicho aprendizaje. En la parte izquierda de la Fig. 1, aparece la influencia de tres canales perceptivos (auditivo, visual y cenestésico o kinestésico) relacionándolos con la actividad del estudiante, activa o pasiva. En parte de la derecha de la figura, se muestran las actividades que pueden desarrollarse junto con el porcentaje de retención, después de 24 horas, en función de la actividad o tarea.

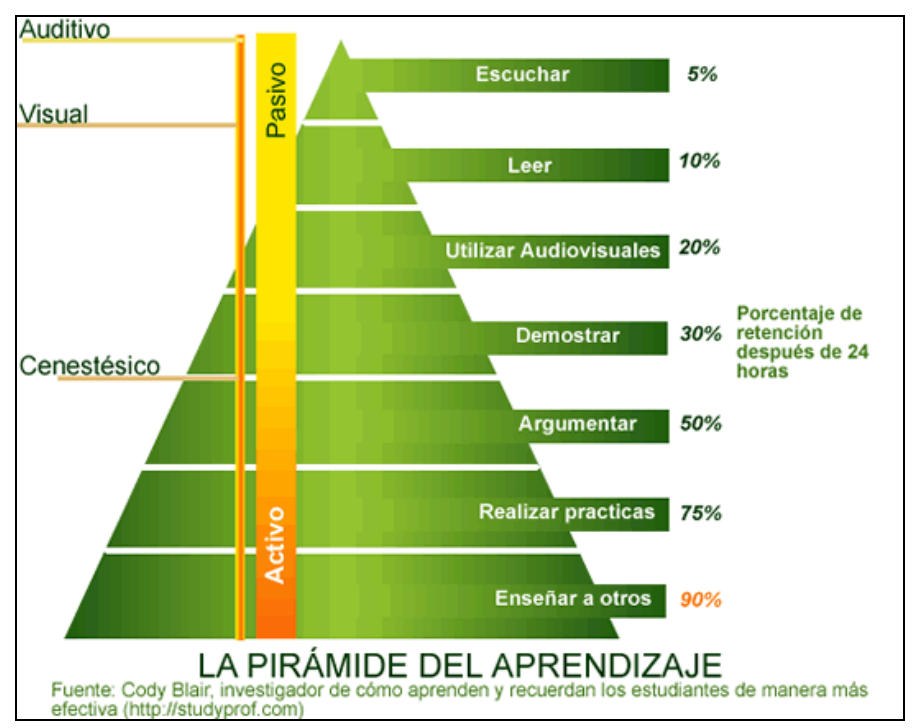

Fig. 1 Pirámide del aprendizaje de Blair

Como puede apreciarse, el porcentaje de retención en aquellas actividades que solo suponen escuchar al profesor es de un 5\%. Siendo estas actividades sumamente pasivas, son necesarias en muchos casos. Para hacerlas más efectivas, es recomendable combinarlas con preguntas, para que el alumno recapacite y exponga, por sí mismo, aquello que ha entendido. Para ello puede elaborar, por ejemplo, esquemas o mapas conceptuales.

La lectura también constituye una actividad pasiva y supone una retención del $10 \%$. No obstante constituye una actividad imprescindible dado que la comprensión lectora es fundamental para analizar guías, manuales, protocolos, o simplemente por el placer que puede aportar leer un texto. La lectura puede complementarse con actividades más dinámicas, como subrayar, hacer un resumen o contestar a una batería de preguntas que reflejen lo aprendido. 
La utilización de medios audiovisuales permite retener un 20\%. Dichos medios son muy valorados por el alumnado puesto que los estudiantes son muy receptivos a las tecnologías interactivas. Téngase en cuenta que el uso de vídeos tutoriales de internet está bastante generalizado, sea para preparar exámenes, para repasar lo aprendido o para abordar nuevos contenidos en el caso de no poder asistir a clase. Por otra parte no todos los estudiantes tienen el mismo ritmo de aprendizaje, y la posibilidad de pausar y rebobinar, que ofrecen los vídeos, es de gran ayuda (Bergamnn y Sams, 2015). Los medios audiovisuales son una buena herramienta para captar la atención del estudiante, pero es necesario realizar posteriormente actividades para ampliar y profundizar sobre el tema en cuestión.

La demostración (simulaciones y experimentos) supone una retención del 30\%, y puede complementarse, por ejemplo, con actividades colaborativas. Se puede aprovechar la metodología del puzle de Aronson (Aronson et al., 1978,1997) para alguna de estas actividades.

Argumentar supone una tarea compleja que implica que el estudiante practique diversas competencias, como comprender el tema, elegir entre diversas alternativas, posicionarse y saber defender determinada postura ante sus compañeros. En este caso el porcentaje de retención aumenta hasta el 50\%. Este aspecto puede ser utilizado en clase por el profesor, antes de introducir un tema, pidiendo la opinión o una argumentación a sus estudiantes o creando de grupos de discusión.

Parece claro que se aprende más cuando se hace activamente más, es decir, cuando se realiza una práctica, alcanzándose el $75 \%$ de retención en este caso. Se trata de que planteemos a nuestros estudiantes actividades prácticas y ejercicios en clase (resolución de problemas, proyectos, experimentos, análisis de casos, etc.). Estas actividades pueden realizarse en grupos pequeños.

Finalmente, la actividad que facilita más retención, el 90\%, es la de enseñar a otros, puesto que esto supone, primero, tener cierto dominio del tema a explicar y después pensar la mejor forma de expresarlo para lograr la comprensión de los receptores. Para esto pueden plantearse exposiciones en grupo. También en este caso la utilización del puzle de Aronson aporta beneficios en el sentido de que, repartiendo la materia a exponer, finalmente todo el equipo acaba aprendiendo dicha materia

Con el segundo PIME, "Puesta en marcha de diversas experiencias con el enfoque Flipped Teaching en asignaturas de Matemáticas y Física" (convocatoria 2016-2017) se ha aplicado esta metodología en algunas de las asignaturas involucradas en ambos proyectos. En este trabajo se presenta la labor docente realizada desde el curso 2015-2016 en una de ellas, Matemáticas 2.

Matemáticas 2 es una asignatura anual que se imparte en el primer curso del Grado en Ingeniería de Sistemas de Telecomunicación, Sonido e Imagen (GISTSI) del Campus de Gandia de la UPV. Tiene un total de 9 créditos ECTS repartidos en 4.6 ECTS de clases de teoría y práctica de aula, 3 ECTS de clases de seminario de problemas y 1.4 ECTS de prácticas de laboratorio informático, distribuidos en 7 prácticas de dos horas de duración. Las prácticas se realizan con la ayuda del paquete de cálculo $\mathrm{Matlab}^{\oplus}$, y son las siguientes:

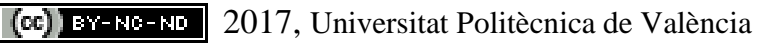


1. Iniciación al Matlab ${ }^{\oplus}$ y sus aplicaciones matemáticas

2. Ecuaciones paramétricas y polares (de los corazones, flores, mariposas...)

3. Ajuste e interpolación

4. Integración aproximada

5. Examen de las prácticas del primer semestre

6. Representación de superficies: forma cartesiana y paramétrica

7. Representación de superficies en forma discreta

Las cinco primeras prácticas se realizan durante el primer semestre. En la nota de la asignatura, un $20 \%$ corresponde a la nota de prácticas, un $70 \%$ a diversas pruebas parciales de teoría y problemas y un $10 \%$ al portafolio. A pesar de no ser muy alto el porcentaje de la nota asignado a prácticas (20\%), los resultados de cursos anteriores indican que una cuarta parte del alumnado aprueba la asignatura gracias a las prácticas.

Según un informe del Vicerrectorado para el Desarrollo de las Tecnologías de la Información y de las Comunicaciones realizado durante el curso 2015-16, los estudiantes creen que es más conveniente aplicar el FT en asignaturas prácticas. Este hecho se ha tenido en cuenta en nuestra asignatura a la hora aplicar esta metodología. En este trabajo nos centramos en el proceso llevado a cabo para pasar de las prácticas tradicionales a las invertidas. Cabe mencionar que, de forma puntual, se ha aplicado el FT en clases de teoría de aula, recurriendo al llamado Micro Flip Teaching (MFT). El MFT es una variante del FT, pero aplicado en este caso sólo en partes de algún que otro tema y no necesariamente en todo un tema o una asignatura. Existen estudios que avalan la repercusión del MFT en la mejora del aprendizaje y en la consolidación de conocimientos (Fidalgo, 2017).

Existen muchas herramientas que facilitan poder implementar la metodología FT. En nuestro caso la herramienta Lessons de la plataforma de la UPV, PoliformaT, ha sido muy util para crear recursos de forma sencilla y atractiva permitiendo integrar otras utilidades de PoliformaT. Además, gracias a que los Lessons permiten la interacción con los usuarios, hemos podido ir perfeccionando las unidades creadas a partir de la opinión de los estudiantes.

\section{Objetivos}

Uno de los objetivos docentes que llevó a incorporar el FT en las prácticas de laboratorio fue aprovechar las clases presenciales para la realización de un trabajo en grupo. Aunque, anteriormente, al alumnado también se le exigía la realización de un trabajo similar, este se hacía posteriormente a la sesión de prácticas, en horas no lectivas. Con frecuencia aparecían problemas en los grupos, como incompatibilidad de horarios, falta de compromiso por parte de algún miembro, etc. Al invertir las clases prácticas, las horas presenciales se dedican a la realización del trabajo en grupo, evitándose así muchos problemas, a la vez que se facilita que el profesor pueda estar más pendiente del aprendizaje efectivo. 


\section{Desarrollo de la innovación}

La idea central de la metodología FT es la de dar la vuelta al método de enseñanza común o modelo tradicional. Y eso es lo que se ha hecho con las prácticas informáticas de la asignatura. Durante muchos años hemos ido innovando en la realización de las prácticas informáticas, haciendo uso de diversas metodologías, (Vidal et al, 2012, 2013, 2014). Hasta el curso 2014-2015 el alumno tenía a su disposición un guion en formato pdf para cada una de las prácticas. En la sesión presencial el profesor explicaba el guion y era quien dirigía la práctica. Dichos guiones tenían unas secciones más teóricas y otras, denominadas en su día "Manos a la obra", en la que se ponía en práctica la teoría para resolver determinado problema con la ayuda de Matlab $^{\odot}$. En estas secciones, más prácticas, se trabajaban los comandos necesarios para la resolución del problema en cuestión, y se exigía al alumnado que trabajara sobre los comandos indicados. Por lo tanto, durante las dos horas de cada una de las prácticas, el estudiante se limitaba a escuchar al profesor y a repetir aquello que se indicaba en el guion. En los últimos minutos de la sesión presencial, el profesor explicaba el trabajo en grupo, que los estudiantes debían realizar y entregar en un plazo de diez días. La nota de prácticas se obtenía de la valoración del trabajo, sin examen alguno.

Sin embargo, como se ha indicado anteriormente, surgían problemas entre miembros de los grupos de trabajo. Además, en ocasiones, no existía una clara correlación entre la nota obtenida en las pruebas parciales de determinado estudiante con las de sus prácticas. De hecho, algunos estudiantes sugerían la realización de un examen de prácticas. Para evitar este tipo de situaciones, con la incorporación del FT en las prácticas, se han aprovechado las horas presenciales para la ejecución del trabajo en grupo. De esta manera, el profesor ha podido observar la colaboración real de los miembros del grupo y, además, comprobar la adquisición de ciertas competencias transversales. También se ha añadido una evaluación individual de las prácticas al final de las mismas.

La puesta en práctica de los proyectos de innovación señalados anteriormente, ha supuesto la inversión de las prácticas de la asignatura desde el curso 2015-2016. A continuación explicamos los pasos y la metodología seguidos en el proceso de inversión.

\subsection{Generación y elaboración de material}

En el material Sesión de Clase Inversa realizado por Pilar Aurora Cáceres y Susana Martínez acerca del Proyecto Flip en la UPV, que puede encontrarse en la site Proyecto clase inversa de PoliformaT, se indica la importancia de la selección o elaboración de buenos materiales, con la ayuda de las tecnologías adecuadas, así como también del diseño de actividades de aprendizaje significativas tanto para la sesión no presencial como para el aula. Todo esto lleva implícitas varias fases: la primera es la selección de los contenidos de la materia que realmente son importantes para el aprendizaje del alumnado; la segunda elaborar o seleccionar los materiales que recojan dichos contenidos; finalmente determinar qué contenidos pueden trabajar los alumnos, de forma autónoma, en su casa, con los materiales elaborados/seleccionados, y qué contenidos trabajar presencialmente en el aula.

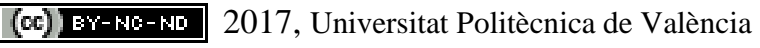

Congreso IN-RED (2017) 
Una de las páginas web españolas en las que se pueden encontrar experiencias y recursos para invertir la clase es "The flipped classroom". En el enlace

http://www.theflippedclassroom.es/un-ejemplo-de-ficha-para-planificar-nuestra-clase-inversa/

se puede encontrar un ejemplo de ficha para planificar una clase inversa, que mostramos en la Figura 2.

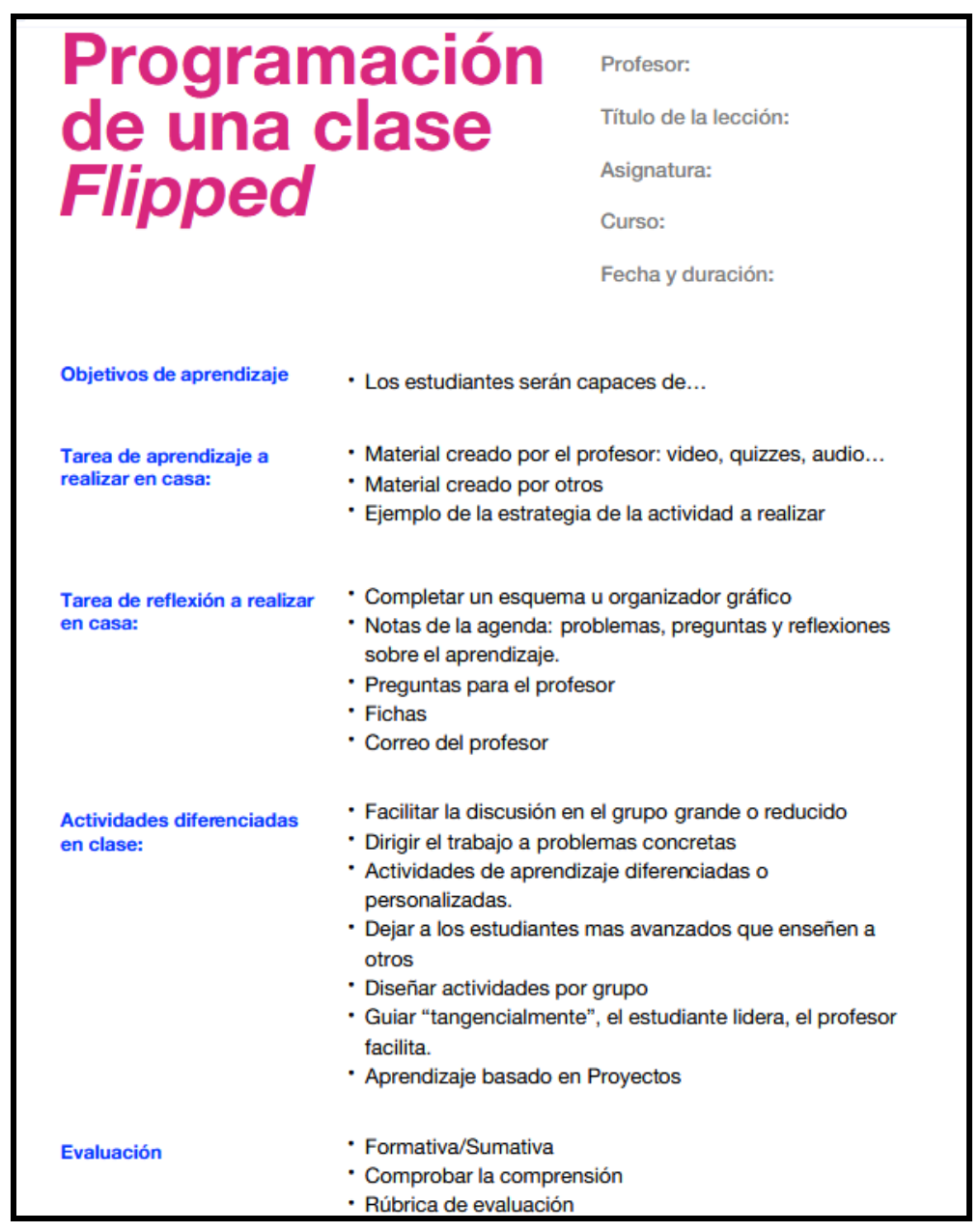

Fig. 2 Ficha de programación de una clase Flip

Lo novedoso de la clase invertida es, sin duda, la parte en la que el estudiante realiza un trabajo autónomo previo a la clase presencial. Esto exige por parte del alumnado dedicación y compromiso, por parte del profesor un cuidadoso trabajo de selección de los materiales de aprendizaje y una distribución adecuada de los tiempos. Sin embargo no es menos importante el trabajo presencial en el aula. Para ello es fundamental que el docente planifique y estructure de forma adecuada el aprendizaje del estudiante (Tourón, Santiago y Díez, 2014). 
Teniendo en cuenta todo lo precedente, en el proyecto "Estudio sobre la aplicación del Flip Teaching en asignaturas de Matemáticas y Física" nos planteamos en su día como objetivo principal, elaborar una amplia colección de fichas de planificación o programación. Se trataba de fichas Flip, similares a la de la Figura 2, que permitieran englobar los objetivos, las actividades y los recursos necesarios para poder realizar cada una de las actividades Flip programadas. En la Figura 3 mostramos una de las fichas elaboradas, la de integración aproximada, correspondiente a la asignatura Matemáticas 2. Pueden obtenerse el resto de fichas de esta asignatura y de otras asignaturas, participantes en los mencionados proyectos de innovación, en

\section{http://flipeandoencampusgandia.simplesite.com}

Las fichas Flip, correspondientes a cada una de las prácticas, facilitaron la estructuración de los recursos necesarios y de las actividades a realizar en cada una de las prácticas.
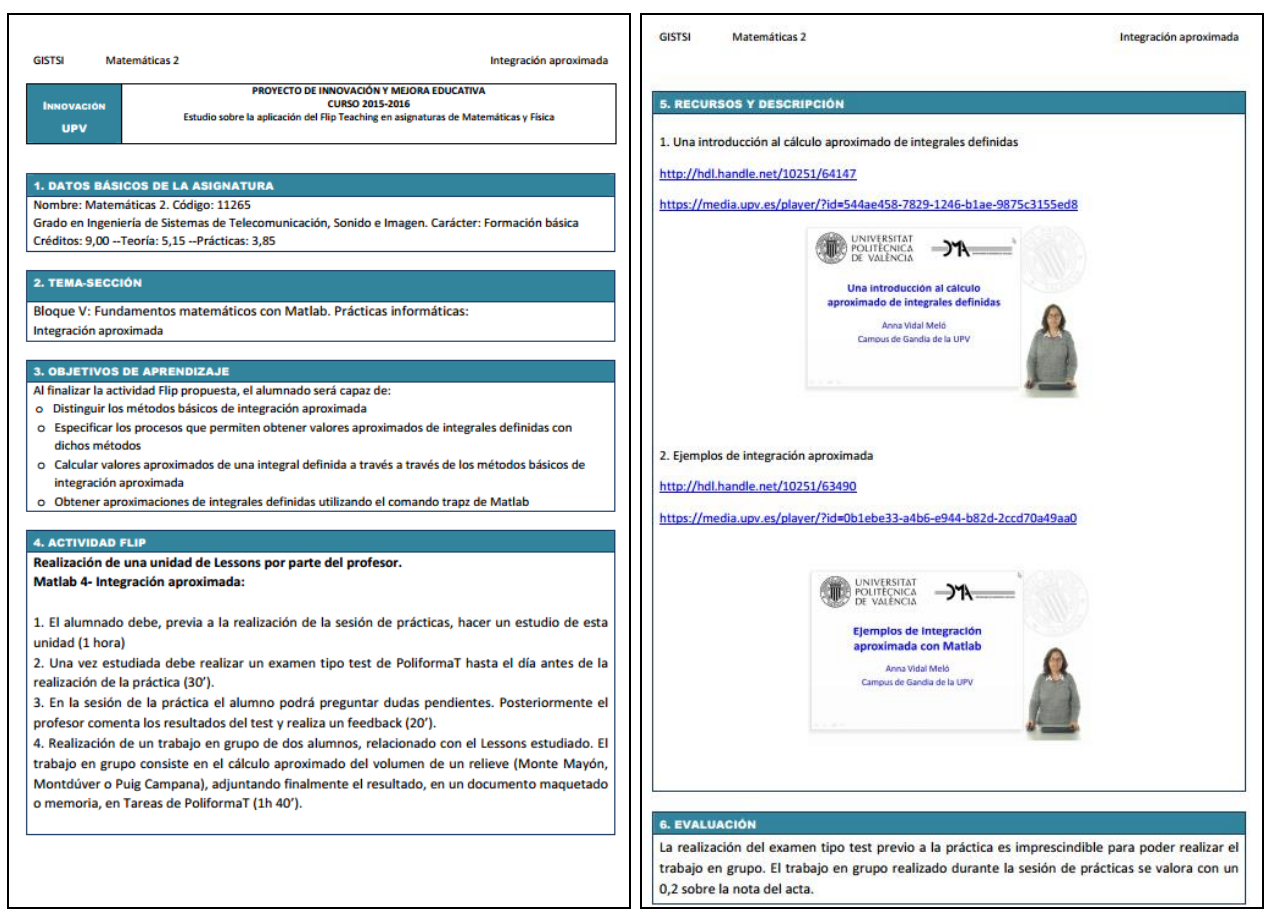

Fig. 3 Ficha Flip correspondiente a la práctica sobre integración aproximada

En cuanto a los recursos necesarios, se inició una búsqueda de recursos multimedia, en particular vídeos, tanto dentro del repositorio institucional de la UPV, Riunet, como en distintas plataformas de la red. Finalmente, aunque algunos vídeos han sido obtenidos de la plataforma YouTube, otros han sido creados exprofeso. En concreto, varios Polimedia y screencast que han pasado a formar parte del repositorio de Objetos de Aprendizaje de la UPV, tras ser aprobados en el Plan de Docencia en Red 2015-2016: Una introducción al ajuste polinómico de datos por mínimos cuadrados, utilizando Matlab; una introducción a la interpolación polinómica con Matlab; una introducción a la interpolación segmentaria con

(cc) EY-NC-ND 2017, Universitat Politècnica de València 
Matlab: comando spline; una introducción al cálculo aproximado de integrales definidas; y, finalmente, ejemplos de integración aproximada.

Una vez elaborada la ficha Flip, pasamos a la creación de las unidades didácticas correspondientes a cada una de las prácticas, salvo de la primera (más adelante indicaremos la razón de ello). Para la creación de unidades didácticas se utiliza la herramienta Lessons de PoliformaT. Los guiones de prácticas son sustituidos por Lessons, lo cual facilita poder enlazar distintos elementos de PoliformaT. Por ejemplo, al final de cada Lesson insertamos un enlace a un examen PoliformaT, con dos finalidades. La primera es que el estudiante compruebe si ha adquirido los conocimientos necesarios para poder llevar a cabo con éxito el trabajo que se le exigirá en la sesión presencial. La segunda es que esto permite controlar qué estudiante ha estudiado el material correspondiente y el nivel de comprensión alcanzado. La fecha de cierre del examen es el día anterior a la sesión presencial de la práctica, lo cual permite hacer un análisis de la situación antes de la sesión presencial. Se aprovecha la posibilidad que tiene la herramienta Lessons para elaborar cuestionarios y poder preguntar a los estudiantes su opinión sobre el contenido generado, como se muestra en la Figura 4.

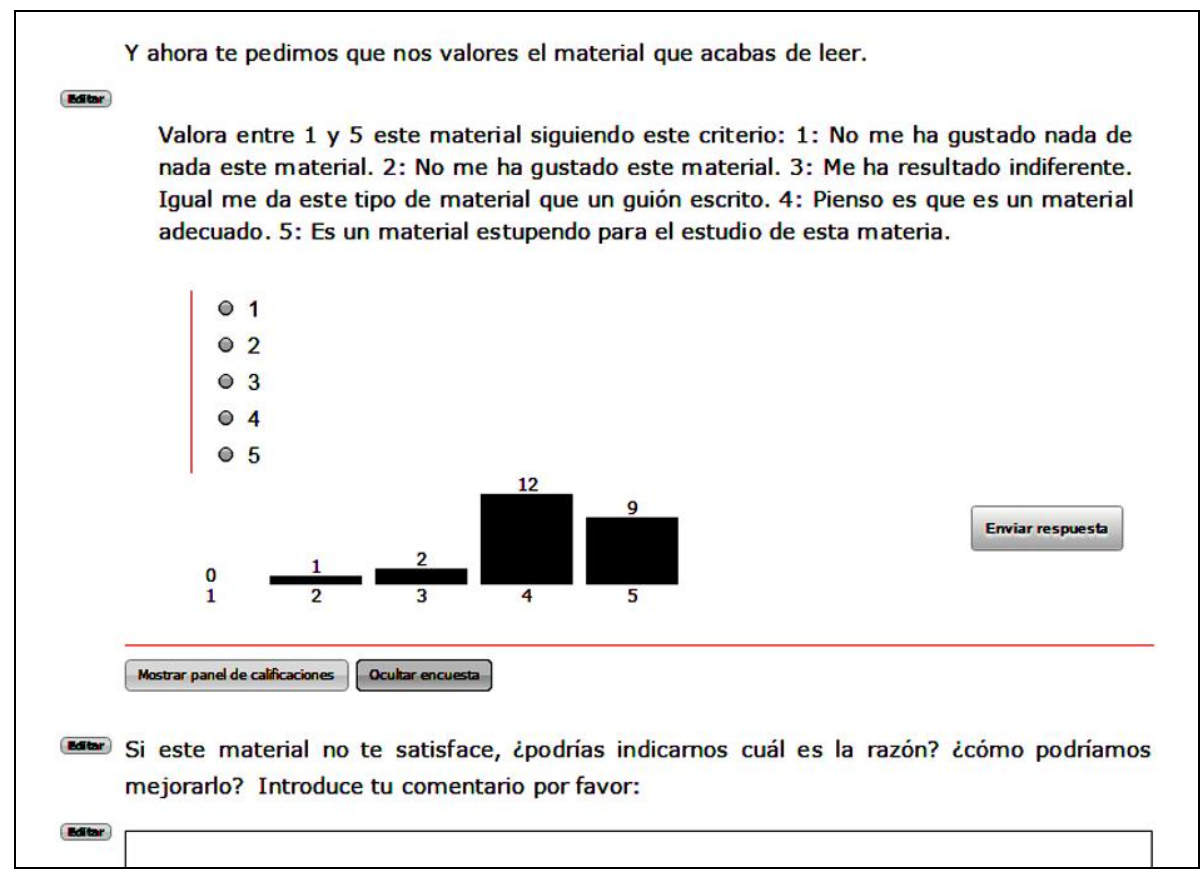

Fig. 4 Cuestionario sobre el contenido del Lessons

En uno de los Lessons, en el que se insertaron varios Polimedia, un par de estudiantes indicaron la necesidad de poder tener acceso a las diapositivas de los vídeos para poder leer los contenidos tranquilamente y poder consultarlos más tarde en caso de necesidad. Según indicaban, sin disponer de las diapositivas resultaba pesado estudiar los contenidos y tenían que pausar el vídeo continuamente. A partir de esas observaciones, en los siguientes Lessons, antes del Polimedia correspondiente, había un hipervínculo que permitía imprimir 
las transparencias, y por lo tanto seguir mejor la explicación del vídeo. Últimamente, y de nuevo al considerar las observaciones de los estudiantes en los Lessons, también se ha insertado un enlace al Foro de PoliformaT, para que puedan resolver aquellas dudas que puedan surgir a medida que avanzan en el estudio.

Por último, cabe señalar que antes de la sesión presencial se elabora, utilizando la aplicación Tareas de PoliformaT, la actividad del trabajo en grupo que deben realizar durante dicha sesión. La Tarea lleva adjunto un fichero con información y, en su caso, otros posibles ficheros que pueden servir de plantilla para la memoria del trabajo (Figuras 5 y 6 ). La fecha de apertura de la Tarea es la de inicio de la sesión presencial.

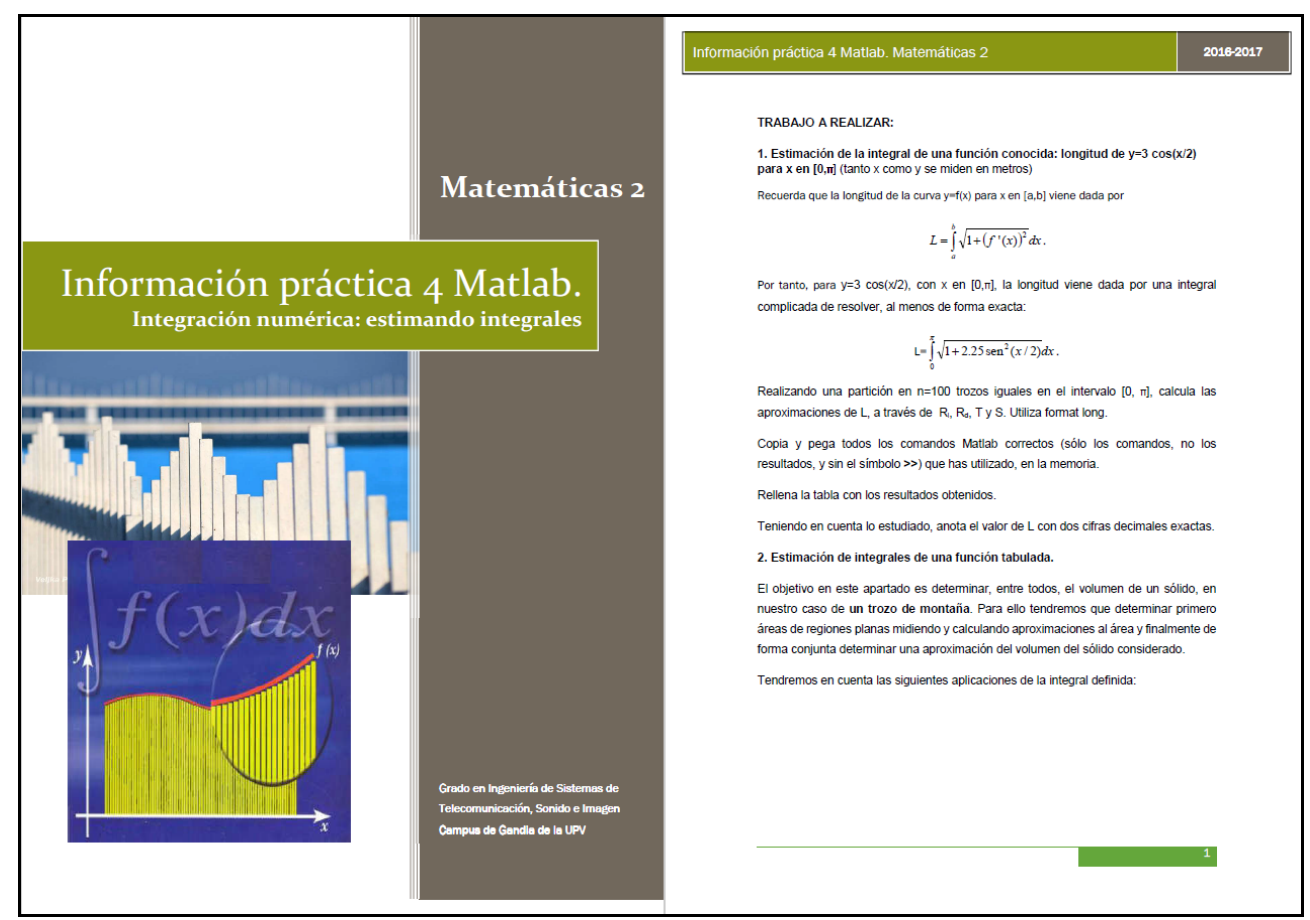

Fig. 5 Primeras hojas del documento informativo correspondiente al trabajo en grupo a realizar

\subsection{Desarrollo de la sesión práctica}

Al inicio de la sesión práctica se resuelven, en su caso, las posibles dudas que plantean los estudiantes para, posteriormente, pasar a comentar los resultados del examen PoliformaT del final del Lesson. Para esto, es muy útil la herramienta Estadísticas del menú Exámenes ya que los propios estudiantes pueden apreciar en qué preguntas han tenido más dificultad. Aquellas preguntas con peores resultados se explican de forma detallada para proporcionar el feedback correspondiente.

En algunos Lessons se aconseja al estudiante la realización de un resumen, necesario para la realización posterior del trabajo. Durante la sesión presencial de prácticas se comprueba qué estudiantes han realizado dicha labor.

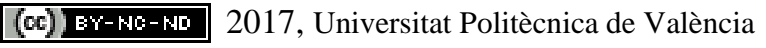




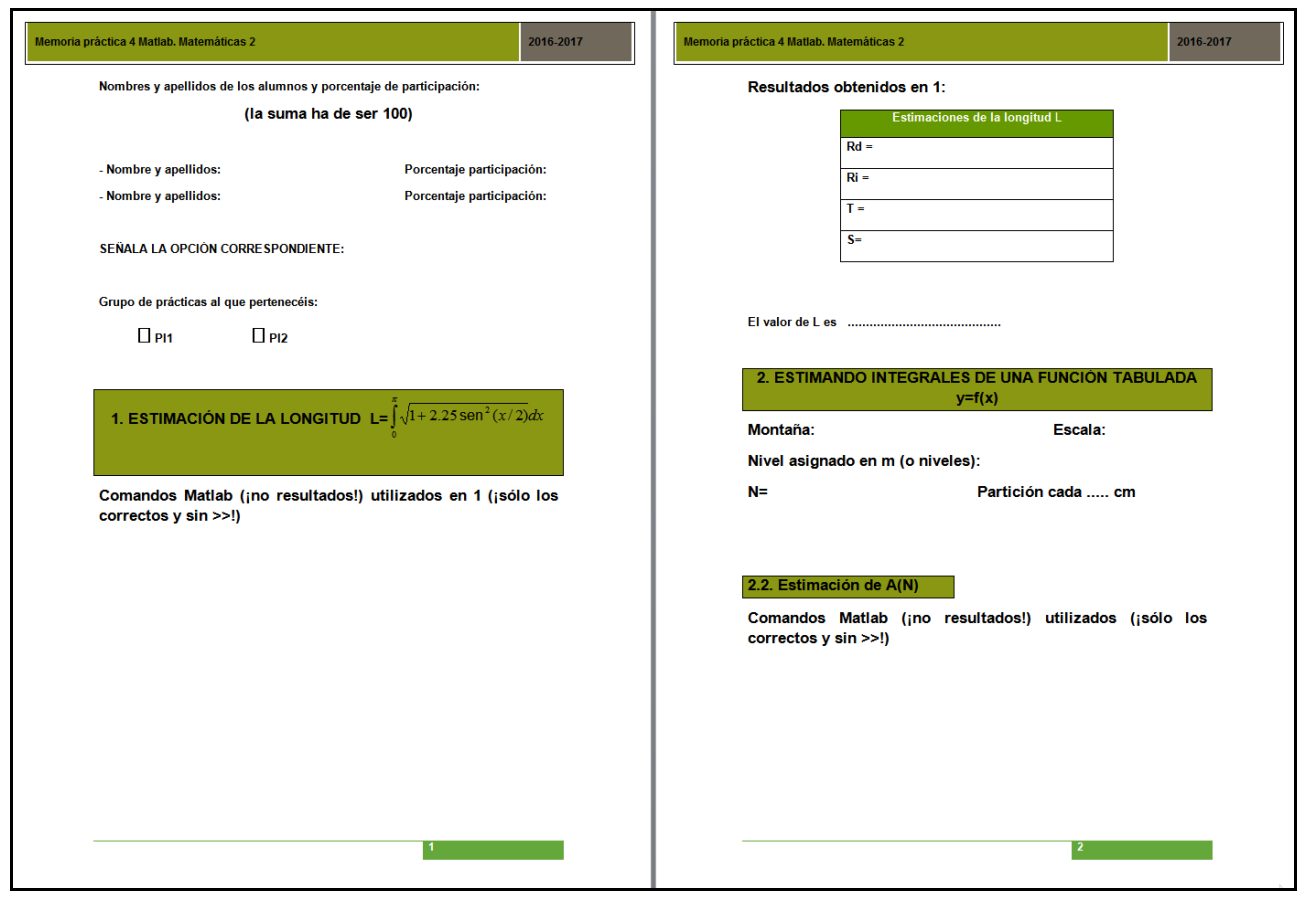

Fig. 6 Primeras hojas la plantilla de una de las memorias a entregar

A continuación se forman los grupos de trabajo, de dos o tres alumnos. Posteriormente se procede a explicar el trabajo o proyecto a realizar, utilizando como apoyo el documento informativo disponible en Tareas de PoliformaT. Son variados los proyectos que se realizan en dichas prácticas (Vidal et al., 2012, 2013, 2014). Durante el resto de la sesión, los estudiantes trabajan en el proyecto planteado, que en ocasiones es un trabajo colaborativo entre grupos. En estos casos se ha utilizado el programa iTALC (Enseñanza inteligente y aprendizaje con computadoras) en el aula. Se trata de un software libre que permite, entre otras cosas, ver lo que sucede en cada uno de los equipos del aula y proyectar alguno de ellos, favoreciendo el trabajo colaborativo. Finalmente, los estudiantes deben adjuntar, en la Tarea creada al efecto, el archivo o archivos correspondientes al trabajo realizado.

Llegados a este punto, cabe plantearse cómo afrontar un problema importante: ¿Qué hacer con los estudiantes que no completan la fase inicial (no presencial) de estudiar el Lessons y realizar el examen tipo test?. En el blog Profesor 3.0 (Prieto, 2016) se dan soluciones para este punto débil del Flip, aconsejándose la realización de tareas con una metodología que permita comprobar el estudio previo y que motive a los estudiantes a estudiar los materiales. Las herramientas Exámenes y Estadísticas de PoliformaT permiten controlar el seguimiento de los contenidos en la fase no presencial. Los estudiantes que incumplen son ubicados en la última fila del aula, separados del resto de estudiantes. Su trabajo durante la sesión de prácticas consiste en realizar la tarea que deberían haber realizado en casa (preparación del Lessons) y la realización del examen previo, debiendo obtener, en este caso, al menos un 6 para poder realizar el trabajo en grupo y así obtar a la puntuación de la práctica. A estos alumnos se les daba un día de plazo para la realización del trabajo. En 
general, ninguno de estos alumnos entregó el trabajo en tiempo y forma en la primera práctica FT. Pero la experiencia sirvió de motivación para que en las siguientes prácticas estos alumnos se adaptaran a la nueva metodología.

\section{Resultados}

Durante el curso 2015-2016 (metodología FT) se ha notado una mejora en las notas de prácticas respecto a las del curso anterior (metodología tradicional):

- Durante el curso 2014-2015, el porcentaje de alumnos con nota superior o igual a 5 fue del 64.3\%, mientras que en el curso 2015-2016 aumentó hasta el 76.8\%.

- La nota media de los alumnos con nota igual o superior a 5 durante el curso 20142015 fue de 7.8, valor que se incrementó hasta el 8.6 en el siguiente curso.

Otro de los resultados positivos está relacionado con la calidad del material creado con Lessons. En cada uno los Lessons les pedimos a nuestros estudiantes que hicieran una valoración, obteniéndose muy buenos resultados, como se refleja en la Figura 7. La escala utilizada fue: 1: No me ha gustado nada de nada este material; 2: No me ha gustado este material. 3: Me ha resultado indiferente. Igual me da este tipo de material que un guion escrito; 4: Pienso que es un material adecuado; 5: Es un material estupendo para el estudio de esta materia.
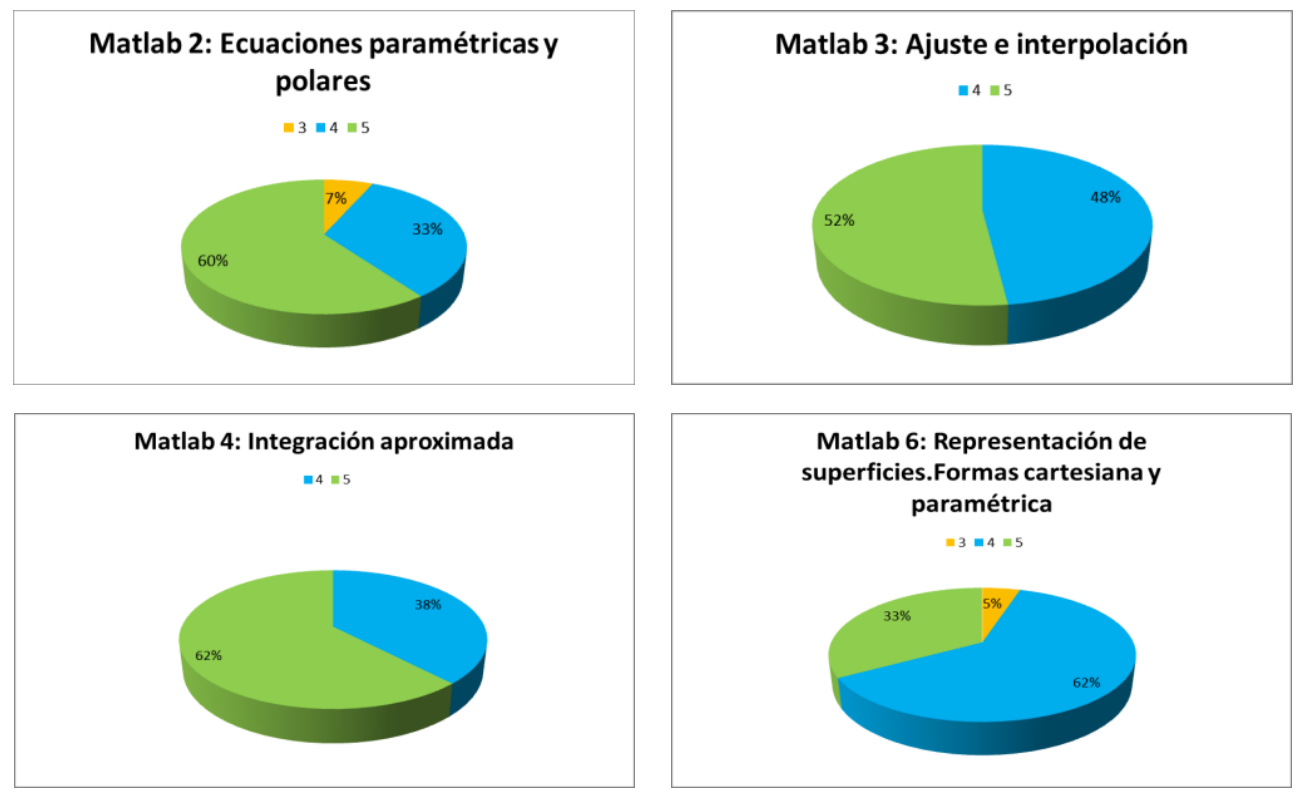

Fig. 7 Opinión del alumnando acerca de los Lessons

La primera práctica de la asignatura sigue la metodología tradicional, detallada en el apartado 2 sobre el desarrollo de la innovación, como una continuación del sistema de aprendizaje que traen los estudiantes como bagaje. Son estudiantes de primer curso que acaban de entrar en la universidad, no adaptados todavía al funcionamiento de las clases, a

(c)) BY-NC-ND 2017, Universitat Politècnica de València

Congreso IN-RED (2017) 
asignaturas con distintos métodos de evaluación, etc. Una vez realizada esta primera práctica se les habla de lo que es el Flipped Teaching y cómo van a desarrollarse las prácticas posteriores. Al final del primer semestre del curso 2016-2017, aprovechando que el estudiante ya puede comparar entre la metodología tradicional, utilizada en la primera práctica, y la metodología FT utilizada en el resto de prácticas, pasamos a los estudiantes una encuesta, similar a la que durante dos años ha sido utilizada por la UPV, para conocer la valoración de los alumnos en lo que respecta a la metodología empleada. Hemos comparado estos resultados con los que aparecen en el informe del Vicerrectorado de Estudios, Calidad y Acreditación de la Universitat Politècnica de València sobre la evaluación del Flip Teaching durante el curso 2015-2016. Este estudio fue realizado para 201 asignaturas y 233 profesores de 13 centros distintos de la UPV. En las Figuras 8, 9 y 10 se comparan los resultados obtenidos con nuestros estudiantes con los que aparecen en el citado informe, apreciándose en nuestro caso unas valoraciones similares o superiores.

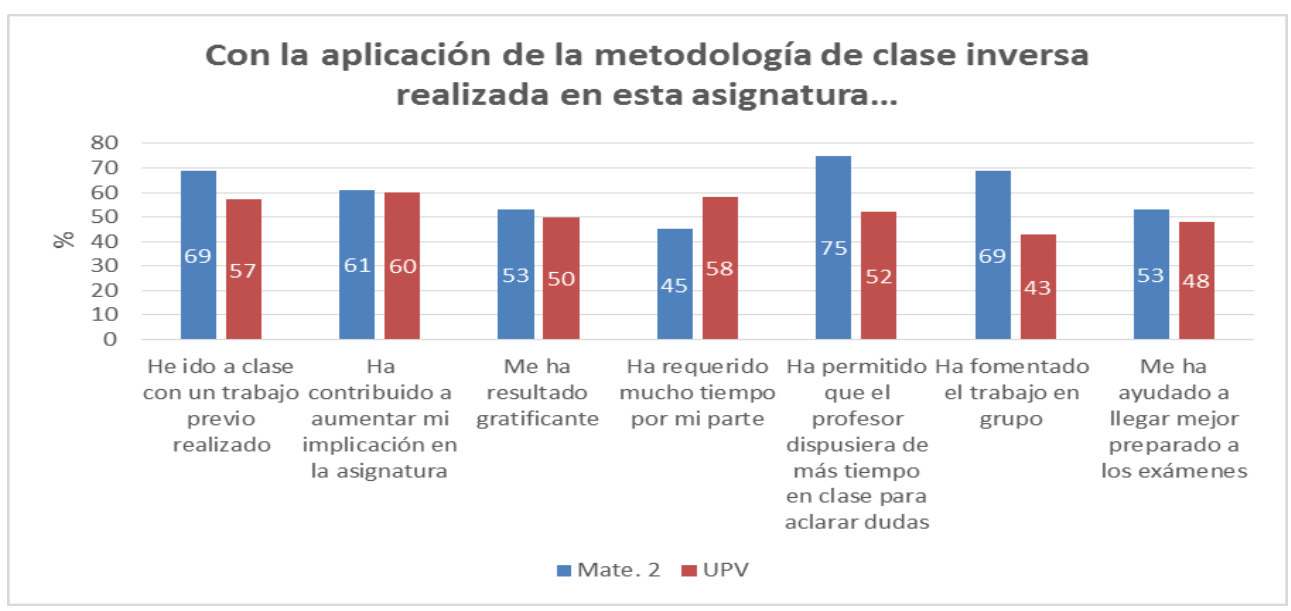

Fig. 8 Opinión sobre la aplicación de la clase inversa

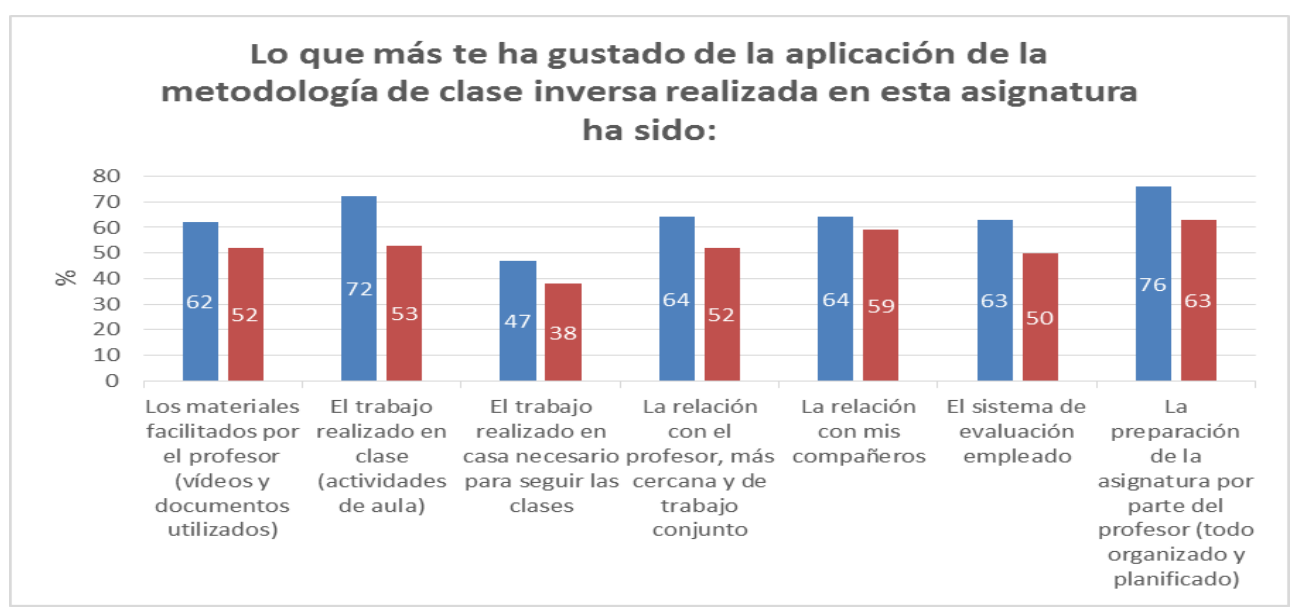

Fig. 9 Lo que más les ha gustado de esta metodología 


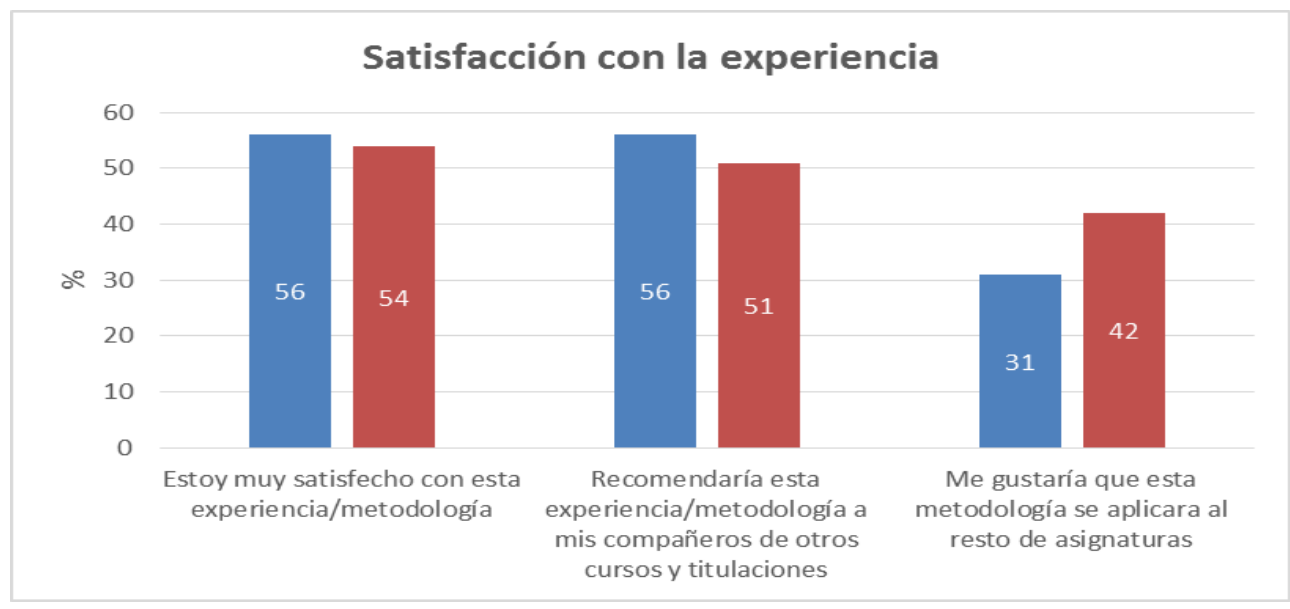

Fig. 10 Satisfacción con la experiencia

En la encuesta, pedimos además a nuestros estudiantes que indicasen al menos dos aspectos positivos y dos negativos a destacar de la metodología FT. Entre los aspectos positivos destaca el que esta metodología les ayuda a llevar la asignatura al día, que aprenden más y mejor, la accesibilidad al material y el trabajo en grupo. Entre los aspectos negativos destaca el que la metodología FT les supone más trabajo, seguido de que se echa en falta la explicación "en directo" del profesor y que seguir el ritmo les crea cierta sensación de ansiedad.

\section{Conclusiones}

El FT no es un método para que el estudiante estudie a su antojo o trabaje sólo. Requiere que el docente planifique y estructure de forma adecuada el aprendizaje del estudiante, y que se controlen al máximo todas y cada una de las fases de esta metodología, destacando en particular la fase no presencial. El FT requiere programación previa, y la elaboración y/o búsqueda de recursos que implica un gran esfuerzo para el docente. En la encuesta, los estudiantes han valorado bien la preparación de la asignatura por parte del profesor (76\%) así como el trabajo realizado en clase (72\%), al que también señalan como uno de los ítems positivos. El poder terminar y entregar el trabajo en la misma sesión de prácticas puede que hay influido también en esta valoración. Respecto a los aspectos negativos, fundamentalmente la ansiedad y el aumento de la carga de trabajo, cabe señalar que se trata de estudiantes de primer curso, acostumbrados a estudiar un tiempo relativamente corto antes de cada examen. A muchos estudiantes no les gusta que se les planifique el estudio y prefieren ir a su ritmo. El FT requiere un cambio en la mentalidad y en la rutina de los estudiantes. A pesar de todo, reconocen que el FT les ayuda a preparar mejor las asignaturas y a ir más al día.

Haber adoptado el FT en nuestras prácticas ha supuesto una mejora en las notas de prácticas respecto a las del curso anterior y los estudiantes han valorado bien el material proporcionado. Teniendo en cuenta los resultados aportados, pensamos que la elección e implementación de la metodología FT ha sido adecuada.

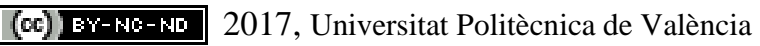




\section{Agradecimientos}

Este trabajo se ha realizado gracias al apoyo del Vicerrectorado de Estudios, Calidad y Acreditación de la UPV al Proyecto PIME 2016-2017 "Puesta en marcha de diversas experiencias con el enfoque Flipped Teaching en asignaturas de Matemáticas y Física".

\section{Referencias}

ARONSON,E., BLANEY, N., STEPHIN, C., SIKES, J. \& SNAPP, M. (1978). The Jigsaw Classroom. Beverly Hills, California. Sage Publications.

ARONSON, E., PATNOE, S. (1997). The Jigsaw Classroom, Building Cooperation in the Classroom. United States. Longman (second edition).

BERGMANN, J.SAMS, A. (2015). Dale la vuelta a tu clase: Lleva tu clase a cada estudiante, en cualquier momento y cualquier lugar. SM.

CACERES GONZALEZ., P.A., MARTINEZ NAHARRO, S. " Proyecto flip teaching en la UPV: cómo invertir nuestras clases a través de las herramientas tecnológicas”. ICE-ASIC.

FIDALGO-BLANCO, A., MARTINEZ-NUÑEZ, M., BORRAS-GENE, O., \& SANCHEZ-MEDINA, J. J. (2017). "Micro flip teaching-An innovative model to promote the active involvement of students" en Computers in Human Behavior, 72, 713-723.

SANTIAGO, R. Y DIEZ, A. (2015) The flipped classroom. <http://www.theflippedclassroom.es/> [Consulta: 15 de marzo 2017]

PRIETO MARTÍN, A. (2016) “¿Funciona el modelo flipped con alumnos españoles? Aspectos críticos para lograr el éxito de los métodos de fomento del estudio previo y flipped classroom y flipped learning)“ en Profesor 3.0, 1 de agosto 2016. <http://profesor3punto0.blogspot.com.es/2015/04/claves-para-el-exito-de-los-metodos-de.html> [Consulta: 15 de marzo 2017]

TOURÓN, J., SANTIAGO, R. y DIEZ, A. (2014). The Flipped Classroom: Cómo convertir la escuela en un espacio de aprendizaje. Grupo Océano. Digital-Text.

VIDAL MELÓ, A., ROIG SALA, B., ESTRUCH FUSTER, V.D., BOIGUES PLANES, F.J., DEL REY, R., Y ALBA, J. (2012). "Rompiendo con la rutina: dos experiencias matemáticas con el puzle de Aronson" en XX CUIEET. Las Palmas de Gran Canaria. 12 páginas.

VIDAL MELÓ, A., ROIG SALA, B., ESTRUCH FUSTER, V.D., Y BOIGUES PLANES, F. J. (2013). "Una yincana para descubrir y generar curvas en un trabajo cooperativo", en Actas del XXI CUIEET. Valencia. 950-961.

VIDAL MELÓ, A., ROIG SALA, B., ESTRUCH FUSTER, V.D., BOIGUES PLANES, F. J., DEL REY, R., ALBA, J. y SAPENA PIERA, A. (2014). "El poder de la colaboración”, en Actas de las Jornadas de Innovación Educativa y Docencia en Red. 689-703.

VIDAL MELÓ, A., ESTRUCH FUSTER, V.D., BOIGUES PLANES, F. J., DEL REY, R., ALBA, J., ROIG, B. Y TORREGROSA CABANILLES, C. (2016). "Flipped Teaching: una metodología en construcción”, en Actas de las Jornadas de Innovación Educativa y Docencia en Red. 375 - 389.

VIDAL, A. (2017) Proyecto de Innovación educativa: Flipped Teaching. $<$ http://flipeandoencampusgandia.simplesite.com> [Consulta: 15 de marzo 2017]. 